\title{
People diagnosed with schizophrenia retain the diagnosis long term
}

Veen ND, Selten JP, Schols D, et al. Diagnostic stability in a Dutch psychosis incidence cohort. Br J Psychiatry 2004; 185:460-4.

\section{What follow up diagnoses are made in people with psychotic disorders?}

METHODS

$\square$

Design: Prospective longitudinal study.

(3)

Setting: The Netherlands; enrolment from April 1997 to October 2001

क्री

Population: 168 people living in The Hague, Netherlands who consulted a doctor for the first time about a suspected psychotic disorder and received an extensive diagnostic protocol. Exclusions: psychotic disorders induced by substance abuse.

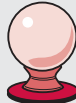

Prognostic factors: Diagnoses were compared at the start of the study and after 2.5 years.

Outcomes: Diagnoses of schizophrenia, major depressive and bipolar disorder with psychotic features, and non-organic or organic psychotic disorders.

$\square \square$

Follow up period: 2.5 years.

\section{MAIN RESULTS}

Most people (120/168, 71\%) were given the same diagnosis after 2.5 years as at the cohort's inception. The highest repeat diagnosis rate was for schizophrenic disorders (92/101, 91\%), compared with $14 / 21(67 \%)$ for psychotic mood disorders and 14/46 (30\%) for other non-organic psychotic disorders. The proportion of people diagnosed with schizophrenic disorders rose from 101/168 (60.1\%) at study's start to $125 / 168(74.4 \%)$ at 2.5 years. The proportion of people with organic psychotic disorder diagnosis rose from 0/168 (0\%) at study start to $5 / 168(3.0 \%)$ at 2.5 years but it is not clear if this diagnostic category was available at first diagnosis. The proportion of people diagnosed with psychotic mood disorder and other non-organic psychotic disorders was lower at 2.5 years compared with study start (proportion of people with psychotic mood disorder diagnosis: 21/168 $(12.5 \%)$ at study start $v 17 / 168(10.1 \%)$ at 2.5 years; proportion of people with other non-organic psychotic disorder diagnosis: 46/168 $(27.4 \%)$ at study start $v 21 / 168(12.5 \%)$ at 2.5 years $)$.

\section{CONCLUSIONS}

The stability of a diagnosis of schizophrenia or of psychotic mood disorder is high over 2.5 years. The apparent prevalence of schizophrenia rises over time in people with psychotic disorders.

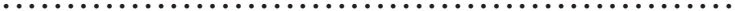

For correspondence: Dr Natalie D Veen, Department of Psychiatry, University Medical Centre, Utrecht, PO Box 85500, 3508 GA Utrecht, the Netherlands; n.veen@pysch.azu.nl

Sources of funding: none declared.

\section{NOTES}

The authors infer from these data that their initial diagnostic protocol leads to stable diagnoses for each of the four categories of psychotic disorder. However, this interpretation assumes there is no underlying change in prevalence and that the two diagnostic assessments were both correct. It is not clear if these assumptions hold, so the conclusions should be treated with caution. The relative contributions to diagnoses of the diagnostic protocols and clinical judgement are also unclear.

\section{Commentary}

7 he number of studies on diagnostic stability within psychiatry is small. This is a problem as diagnostic stability is one criterion for validating psychiatric diagnoses. ${ }^{1}$ Changing diagnoses over time reduces the effectiveness of treatment and impacts upon the outcome for the patient. Recent studies in clinical practice have found a rather poor diagnostic stability of the ICD-10 diagnosis of depressive disorder ${ }^{2}$ and a modest diagnostic stability of bipolar disorder. ${ }^{3}$

The findings of Veen et al have several clinical implications. They show that it is possible, in research settings, to diagnose schizophrenia reliably using a manualised semi-structured interview. The finding that the majority of people who initially presented with delusional disorder or brief psychotic disorder were eventually diagnosed with schizophrenia highlights the need to intensively investigate people presenting with first psychosis. The diagnostic stability of other disorders, such as severe depression and mania, should be investigated in future studies. On a theoretical level, the findings challenge the cross sectional paradigm used in diagnostic systems and emphasises the need to take a longitudinal approach to the diagnostic process.

Lars Vedel Kessing, Professor, MD, DMSc Department of Psychiatry, University Hospital of Copenhagen, Rigshospitalet, Copenhagen, Denmark

1 Robins E, Guze SB. Establishment of diagnostic validity in psychiatric illness: its application to schizophrenia. Am J Psychiatry 1970;126:983-7.

2 Kessing LV. Diagnostic stability in depressive disorder as according to ICD10 in clinical practice. Psychopathology 2005;38:32-7.

3 Kessing LV. Diagnostic stability in bipolar disorder in clinical practise as according to ICD-10. J Affect Disord 2005;85:293-9. 